\title{
Arithmetic of certain hypergeometric modular forms
}

\author{
by \\ Karl Mahlburg and Ken Ono (Madison, WI)
}

1. Introduction and statement of results. If $k \geq 4$ is even, then let $M_{k}$ denote the finite-dimensional $\mathbb{C}$-vector space of weight $k$ holomorphic modular forms on $\mathrm{SL}_{2}(\mathbb{Z})$. As usual, we identify a modular form $f(z)$ with its Fourier expansion

$$
f(z)=\sum_{n=0}^{\infty} a(n) q^{n},
$$

where $q:=e^{2 \pi i z}$. In a recent paper [K-Z], Kaneko and Zagier examined, for even integers $k \equiv 0,4,6,10(\bmod 12)$, the unique normalized modular form $F_{k}(z) \in M_{k}$ that is a solution to the second order differential equation

$$
\theta_{k+2} \theta_{k} F_{k}(z)-\frac{k(k+2)}{144} E_{4}(z) F_{k}(z)=0 .
$$

Here $\theta_{k}$ is the differential operator defined on $f(z) \in M_{k}$ by

$$
\theta_{k} f(z):=q \frac{d}{d q}(f(z))-k E_{2}(z) f(z) / 12 .
$$

For each $4 \leq k \equiv 0,4,6,10(\bmod 12)$, define integers $\delta_{k}, \varepsilon_{k} \in\{0,1\}$ by

$$
4 \delta_{k}+6 \varepsilon_{k}=r,
$$

where $k \equiv r(\bmod 12)$. For such $k$, define the polynomial $\widetilde{F}_{k}(j) \in \mathbb{Q}[j]$ by the identity

$$
F_{k}(z)=\Delta(z)^{[k / 12]} E_{4}(z)^{\delta_{k}} E_{6}(z)^{\varepsilon_{k}} \widetilde{F}_{k}(j(z)) .
$$

Here $j(z)$ denotes the usual $j$-function

$$
j(z)=q^{-1}+744+196884 q+\ldots
$$

2000 Mathematics Subject Classification: Primary 11F11, 33C45.

Key words and phrases: hypergeometric modular forms.

The first author thanks for the generous support of an NSF Graduate Research Fellowship. The second author is supported by NSF grant DMS-9874947, an Alfred P. Sloan Foundation Research Fellowship, a David and Lucile Packard Research Fellowship, an H. I. Romnes Fellowship, and a John S. Guggenheim Fellowship. 
Kaneko and Zagier [K-Z] found beautiful congruences relating these "hypergeometric" modular forms to the loci of supersingular $j$-invariants. More precisely, if $p \geq 5$ is prime, then they proved that

$$
j^{\delta_{p-1}}(j-1728)^{\varepsilon_{p-1}} \widetilde{F}_{p-1}(j) \equiv \prod_{\substack{E / \mathbb{F}_{p} \\ \text { supersingular }}}(j-j(E))(\bmod p) .
$$

The analogous property is enjoyed by the classical Eisenstein series $E_{p-1}(z)$.

These modular forms have further properties in common with the Eisenstein series. A classical theorem of Rankin and Swinnerton-Dyer [R-S] asserts that the zeros $\tau$ of $E_{k}(z)$ are simple. Moreover, if $\tau$ is such a zero, then $j(\tau)$ is real and in the interval $[0,1728]$. In a recent paper $[\mathrm{K}]$, Kaneko observed (Theorem 3 there), thanks to the theory of orthogonal polynomials, that the $F_{k}(z)$ also enjoy these properties.

Here we address analogs of some conjectured properties of the Eisenstein series. If $H / \Gamma$ denotes the usual fundamental domain of $\mathrm{SL}_{2}(\mathbb{Z})$, then it is widely believed that the polynomial

$$
\mathcal{E}_{k}(j)=\prod_{\tau \in H / \Gamma-\{i, \omega\}}(j-j(\tau))^{\operatorname{ord}_{\tau}\left(E_{k}\right)} \in \mathbb{Q}[j]
$$

is irreducible over $\mathbb{Q}$. In fact, it is believed that the Galois group of the splitting field of $\mathcal{E}_{k}(j)$ is the full symmetric group $S_{d(k)}$, where $d(k)$ is the degree of $\mathcal{E}_{k}(j)$. Extensive numerical evidence suggests the following analog.

ConjeCture. If $4 \leq k \equiv 0,4,6,10(\bmod 12)$, then $\widetilde{F}_{k}(j)$ is irreducible over $\mathbb{Q}$. Furthermore, if $\widetilde{F}_{k}(j)$ has degree $d_{k}$, then the Galois group of its splitting field over $\mathbb{Q}$ is $S_{d_{k}}$.

Little is presently known about such questions for Eisenstein series. In particular, it is not known whether infinitely many of the $\mathcal{E}_{k}(j)$ are irreducible over $\mathbb{Q}$. In 1996, Kaneko and Niiho $[\mathrm{K}-\mathrm{N}]$ studied these questions for $\widetilde{F}_{12 n}(j)$ and provided an infinite subclass which are irreducible. Here we provide further results on these questions. We identify several infinite classes of $\widetilde{F}_{k}(j)$ that are irreducible. In addition, we make the observation that many of these $\widetilde{F}_{k}(j)$ have Galois groups which are not subgroups of $A_{d_{k}}$, the alternating group of degree $d_{k}$.

Theorem 1.1. Suppose that $p \geq 5$ is prime, $r \in\{0,4,6,10\}$, and $s$ is a non-negative integer. Furthermore, suppose that $n$ is a positive integer of the form

$$
n= \begin{cases}\frac{p-1}{6} \cdot p^{s} & \text { if } r=0 \text { and } p \equiv 1(\bmod 6), \\ \frac{p^{2}-1}{6} \cdot p^{2 s} & \text { if } r=0 \text { and } p \equiv 5(\bmod 6),\end{cases}
$$




$$
\begin{aligned}
& n= \begin{cases}\frac{20 \cdot 5^{2 s}}{6} & \text { if } r=4 \text { and } p=5, \\
\frac{p-5}{6} & \text { if } r=4, p \equiv 5(\bmod 6) \text { and } p \geq 11, \\
\frac{p^{3}-p}{6} \cdot p^{2 s} & \text { if } r=4, p \equiv 5(\bmod 6) \text { and } p \geq 11,\end{cases} \\
& n= \begin{cases}7^{s} & \text { if } r=6, p=7 \text { and } s \geq 1, \\
\frac{p-7}{6} & \text { if } r=6, p \equiv 1(\bmod 6) \text { and } p \geq 13, \\
\frac{p-1}{6} \cdot p^{s} & \text { if } r=6, p \equiv 1(\bmod 6), p \geq 13 \text { and } s \geq 1, \\
\frac{p^{2}-7}{6} & \text { if } r=6 \text { and } p \equiv 5(\bmod 6), \\
\frac{p^{2}-1}{6} \cdot p^{2 s} & \text { if } r=6, p \equiv 5(\bmod 6) \text { and } s \geq 1,\end{cases} \\
& n= \begin{cases}19 & \text { if } r=10 \text { and } p=5, \\
20 \cdot 5^{2 s} & \text { if } r=10, p=5 \text { and } s \geq 1, \\
220 \cdot 11^{2 s} & \text { if } r=10 \text { and } p=11, \\
\frac{p-11}{6} & \text { if } r=10, p \equiv 5(\bmod 6) \text { and } p \geq 17, \\
\frac{p^{3}-p}{6} \cdot p^{2 s} & \text { if } r=10, p \equiv 5(\bmod 6) \text { and } p \geq 17 .\end{cases}
\end{aligned}
$$

If $k=12 n+r$, then the polynomial $\widetilde{F}_{k}(j)$ is irreducible in $\mathbb{Q}[j]$.

Theorem 1.2. Suppose that $p \geq 5$ is prime, $r \in\{0,4,6,10\}$, and $s$ is a non-negative integer. Furthermore, suppose that $n$ is a positive integer of the form

$$
\begin{aligned}
& n= \begin{cases}\frac{p-1}{6} \cdot p^{s} & \text { if } r=0 \text { and } p \equiv 1(\bmod 12), \\
\frac{p^{2}-1}{6} \cdot p^{2 s} & \text { if } r=0 \text { and } p \equiv 5(\bmod 6),\end{cases} \\
& n= \begin{cases}\frac{20 \cdot 5^{2 s}}{6-5} & \text { if } r=4 \text { and } p=5, \\
\frac{p-p}{6} \cdot p^{2 s} & \text { if } r=4, p \equiv 5(\bmod 12) \text { and } p \geq 17,\end{cases} \\
& n= \begin{cases}\frac{7^{3}}{p-7} & \text { if } r=6, p=7 \text { and } s \geq 1, \\
\frac{p-1}{6} \cdot p^{s} & \text { if } r=6, p \equiv 7(\bmod 12) \text { and } p \geq 19,\end{cases}
\end{aligned}
$$




$$
n= \begin{cases}\frac{220 \cdot 11^{2 s}}{6-11} & \text { if } r=10 \text { and } p=11 \\ \frac{p^{3}-p}{6} \cdot p^{2 s} & \text { if } r=10, p \equiv 11(\bmod 12) \text { and } p \geq 23\end{cases}
$$

If $k=12 n+r$, then $\widetilde{F}_{k}(j) \in \mathbb{Q}[j]$ is irreducible and its Galois group is not a subgroup of $A_{d_{k}}$.

2. Preliminaries. We begin by recalling some important notation. If $n$ is a positive integer, then the Pochhammer symbol $(a)_{n}$ is defined by

$$
(a)_{n}:=a(a+1)(a+2) \ldots(a+n-1) .
$$

If $n=0$, then let $(a)_{n}:=1$. Gauss' ${ }_{2} F_{1}$ hypergeometric functions are defined by

$$
{ }_{2} F_{1}\left(\begin{array}{cc|c}
a & b & x \\
& c & x
\end{array}\right):=\sum_{n=0}^{\infty} \frac{(a)_{n}(b)_{n}}{(c)_{n} n !} \cdot x^{n} .
$$

If $4 \leq k \equiv r(\bmod 12)$, where $r \in\{0,4,6,10\}$, then Kaneko and Zagier provided the following description of the $\widetilde{F}_{k}(j)$ :

$$
\begin{aligned}
\widetilde{F}_{k}(j)= & 1728^{(k-r) / 12}\left(\begin{array}{r}
\left(k+4\left(\delta_{k}-1\right)-6 \varepsilon_{k}\right) / 12 \\
(k-r) / 12
\end{array}\right) \\
& \times{ }_{2} F_{1}\left(\begin{array}{rc|c}
-(k-r) / 12 & (k+r+2) / 12 & j \\
& \left(2 \delta_{k}+2\right) / 3 & \frac{j}{1728}
\end{array}\right) .
\end{aligned}
$$

By $(2.2)$, it follows that $\widetilde{F}_{k}(j)$ is a polynomial of degree $(k-r) / 12$ in $j$.

For our purposes, it suffices to consider, for $r \in\{0,4,6,10\}$ and positive integers $n$, the polynomials given by

$$
{ }_{2} F_{1}\left(\begin{array}{cc|}
-n & n+\beta_{r} \\
\gamma_{r} & x
\end{array}\right) \in \mathbb{Q}[x],
$$

where $\beta_{r}$ and $\gamma_{r}$ are defined by

$$
\beta_{r}:=(r+1) / 6
$$

and

$$
\gamma_{r}:= \begin{cases}2 / 3 & \text { if } r=0,6 \\ 4 / 3 & \text { if } r=4,10\end{cases}
$$

Each ${ }_{2} F_{1}\left(\begin{array}{cc}-n & n+\beta_{r} \\ \gamma_{r}\end{array} \mid x\right)$ is, up to scalar multiple and change of variable, the polynomial $\widetilde{F}_{k}(j)$ where $k=12 n+r$. 
For convenience, we shall study the polynomials $B_{r}(n ; x)$ which are defined by

$$
B_{r}(n ; x):=\sum_{m=0}^{n} c_{r}(n, m) x^{m}:=x^{n} \cdot{ }_{2} F_{1}\left(\begin{array}{cc|c}
-n & n+\beta_{r} \\
\gamma_{r} & \frac{2}{x}
\end{array}\right) .
$$

We choose to work with these polynomials since they are monic. Clearly, $B_{r}(n ; x)$ is irreducible in $\mathbb{Q}[x]$ if and only if $\widetilde{F}_{12 n+r}(j)$ is irreducible in $\mathbb{Q}[j]$. Moreover, they have the same Galois groups. We begin with an elementary description of these polynomials.

Proposition 2.1. Let $n$ be a non-negative integer.

(1) If $r=0$ or 6 , then

$$
B_{r}(n ; x)=\sum_{m=0}^{n} \frac{(-1)^{n-m}\left(\begin{array}{c}
n \\
m
\end{array}\right) \cdot(6 n+r+1)(6 n+r+7) \ldots(6 n+6(n-m)+r-5) x^{m}}{2 \cdot 5 \cdot 8 \cdot \ldots \cdot(3(n-m)-1)} .
$$

(2) If $r=4$ or 10 , then

$$
B_{r}(n ; x)=\sum_{m=0}^{n} \frac{(-1)^{n-m}\left(\begin{array}{c}
n \\
m
\end{array}\right) \cdot(6 n+r+1)(6 n+r+7) \ldots(6 n+6(n-m)+r-5) x^{m}}{4 \cdot 7 \cdot 10 \cdot \ldots \cdot(3(n-m)+1)} .
$$

(Note: Empty products are taken to be 1.)

Proof. These follow from a simple calculation using the fact that

$$
\frac{(-n)_{m}}{m !}=(-1)^{m}\left(\begin{array}{c}
n \\
m
\end{array}\right)
$$

We also require the functions $f_{r}(n), g_{r}(n)$ and $h_{r}(n)$ defined by

$$
\begin{aligned}
& f_{r}(n):=\frac{(12 n+r+1)\left(36 n^{2}+6 r n+6 n+3 \gamma_{r} r-15 \gamma_{r}\right)}{\left(3 n+3 \gamma_{r}\right)(6 n+r+1)(12 n+r-5)}, \\
& g_{r}(n):=-\frac{(12 n+r-5)(12 n+r+1)(12 n+r+7)}{\left(3 n+3 \gamma_{r}\right)(6 n+r+1)(12 n+r-5)}, \\
& h_{r}(n):=-\frac{9 n\left(2 n+(-1)^{r / 2+1}\right)(12 n+r+7)}{\left(3 n+3 \gamma_{r}\right)(6 n+r+1)(12 n+r-5)} .
\end{aligned}
$$

Proposition 2.2. If $r \in\{0,4,6,10\}$, then the $B_{r}(n ; x)$ satisfy the three term recurrence relation

$$
B_{r}(n+1 ; x)=\left(f_{r}(n) x+g_{r}(n)\right) \cdot B_{r}(n ; x)+h_{r}(n) x^{2} \cdot B_{r}(n-1 ; x),
$$

where $B_{r}(-1 ; x)=0$ and $B_{r}(0 ; x)=1$.

Proof. These recurrence relations follow from the definition of the $B_{r}(n ; x)$ and the classical contiguous relation [AAR, p. 100] 


$$
\begin{aligned}
2 b(c-a)(b-a-1) \cdot{ }_{2} F_{1}\left(\begin{array}{cc}
a-1 & b+1 \\
c & x
\end{array}\right)-\left((1-2 x)(b-a-1)_{3}\right. \\
+(b-a)(b+a-1)(2 c-b-a-1)) \cdot{ }_{2} F_{1}\left(\begin{array}{rr|}
a & b \\
c
\end{array} \mid x\right) \\
-2 a(b-c)(b-a+1) \cdot{ }_{2} F_{1}\left(\begin{array}{cc}
a+1 & b-1 \\
c
\end{array} \mid x\right)=0 .
\end{aligned}
$$

One lets $a=-n, b=n+\beta_{r}$ and $c=\gamma_{r}$.

3. The discriminants of the $B_{r}(n ; x)$. Here we compute the discriminants of many of the $B_{r}(n ; x)$. For convenience, we let

$$
D_{r}(n):=\text { discriminant of } B_{r}(n ; x) \text {. }
$$

We express these discriminants in terms of $n, r$, the constant terms of $B_{r}(s ; x)$ for $1 \leq s \leq n$, the value $B_{r}(n ; 2)$, and the recurrence functions $h_{r}(1), h_{r}(2), \ldots, h_{r}(n-1)$.

ThEOREM 3.1. If $r \in\{0,4,6,10\}$ and $n \geq 1$ is an integer for which $B_{r}(n ; 1) \neq 0$, then

$$
D_{r}(n)=(-1)^{n(n-1) / 2}\left(\frac{n\left(n-\gamma_{r}+\beta_{r}\right)}{2 n+\beta_{r}-1}\right)^{n} \cdot \frac{c_{r}(n, 0)}{B_{r}(n ; 2)} \prod_{j=1}^{n-1} h_{r}(j)^{j} c_{r}(j, 0)^{2} .
$$

To prove Theorem 3.1, we begin with a lemma on the derivatives of ${ }_{2} F_{1}$ hypergeometric functions at their zeros.

LEMma 3.2. Suppose that $\alpha \notin\{0,1\}$ is a zero of ${ }_{2} F_{1}\left(\begin{array}{rr}a & b \\ c\end{array} \mid x\right)$. If $a+1 \neq b$, $c \neq 0$ and $b \neq c$, then

$$
{ }_{2} F_{1}^{\prime}\left(\begin{array}{ll|l}
a & b & \\
& c & \alpha
\end{array}\right)=-\frac{a(c-b)}{\alpha(\alpha-1)(a-b+1)} \cdot{ }_{2} F_{1}\left(\begin{array}{cc|c}
a+1 & b-1 \\
c
\end{array} \mid \alpha\right) .
$$

Proof. To prove the lemma, we require the following two facts [AAR, pp. 95-96]:

$$
\begin{aligned}
{ }_{2} F_{1}\left(\begin{array}{ll}
A & B \\
& C
\end{array} \mid x\right)= & (1-x) \cdot{ }_{2} F_{1}\left(\begin{array}{rr}
A+1 & B \\
& C
\end{array} \mid x\right) \\
& +\frac{(C-B) x}{C} \cdot{ }_{2} F_{1}\left(\begin{array}{rc}
A+1 & B \\
C+1
\end{array} \mid x\right), \\
(3.3) \quad x \cdot{ }_{2} F_{1}^{\prime}\left(\begin{array}{ll}
A & B \\
& C
\end{array} \mid x\right)= & A \cdot{ }_{2} F_{1}\left(\begin{array}{rr}
A+1 & B \\
& C
\end{array}\right)-A \cdot{ }_{2} F_{1}\left(\begin{array}{ll}
A & B \\
& C
\end{array}\right) . x
\end{aligned}
$$

By (3.3), where $A=a, B=b$ and $C=c$, we find that

$$
{ }_{2} F_{1}^{\prime}\left(\begin{array}{cc|c}
a & b & \alpha \\
& c & \alpha
\end{array}\right)=\frac{a}{\alpha} \cdot{ }_{2} F_{1}\left(\begin{array}{ll|l}
a+1 & b & \alpha \\
& c &
\end{array}\right) \text {. }
$$


Set $A=b-1, B=a+1$ and $C=c$; then the symmetry of $A$ and $B$ in $(3.2)$ implies that

$$
\begin{aligned}
{ }_{2} F_{1}^{\prime}\left(\begin{array}{ll|}
a & b \\
& c
\end{array} \mid \alpha\right)= & \frac{a}{\alpha(1-\alpha)} \cdot{ }_{2} F_{1}\left(\begin{array}{rr|r}
a+1 & b-1 \\
c & \alpha
\end{array}\right) \\
& -\frac{a(c-a-1)}{c(1-\alpha)} \cdot{ }_{2} F_{1}\left(\begin{array}{cc|c}
a+1 & b \\
& c+1 & \alpha
\end{array}\right) .
\end{aligned}
$$

Replace the last summand by applying (3.2) once more, with $A=a, B=b$ and $C=c$. The fact that $\alpha$ is a zero of ${ }_{2} F_{1}\left(\begin{array}{rr}a & b \\ & c\end{array} \mid x\right)$ implies that

$$
\begin{aligned}
{ }_{2} F_{1}^{\prime}\left(\begin{array}{ll|l}
a & b & \alpha \\
& c & \alpha
\end{array}\right)= & \frac{a}{\alpha(1-\alpha)} \cdot{ }_{2} F_{1}\left(\begin{array}{cc|c}
a+1 & b-1 & \alpha \\
c & \alpha
\end{array}\right) \\
& +\frac{a(c-a-1)}{\alpha(c-b)} \cdot{ }_{2} F_{1}\left(\begin{array}{cc|c}
a+1 & b & \alpha
\end{array}\right) .
\end{aligned}
$$

By (3.4), this last expression is equivalent to the claimed formula for ${ }_{2} F_{1}^{\prime}\left(\begin{array}{ll}a & b \\ & c\end{array} \mid \alpha\right)$.

We obtain the following convenient fact using this lemma.

Proposition 3.3. If $n \geq 1$ and $\alpha \neq 1$ is a zero of $B_{r}(n ; x)$, then

$$
B_{r}^{\prime}(n ; \alpha)=\frac{\alpha n\left(n-\gamma_{r}+\beta_{r}\right)}{\left(2 n+\beta_{r}-1\right)(\alpha-2)} \cdot B_{r}(n-1 ; \alpha) \text {. }
$$

Proof. By definition, we have

$$
B_{r}(n ; x)=x^{n} \cdot{ }_{2} F_{1}\left(\begin{array}{cc|c}
-n & n+\beta_{r} & \frac{2}{x} \\
\gamma_{r} & x
\end{array}\right.
$$

Therefore, it follows that

$$
B_{r}^{\prime}(n ; x)=\frac{n}{x} \cdot B_{r}(n ; x)-2 x^{n-2} \cdot{ }_{2} F_{1}^{\prime}\left(\begin{array}{cc|c}
-n & n+\beta_{r} & \frac{2}{x} \\
\gamma_{r} &
\end{array}\right) .
$$

Since $\alpha \neq 0$ (i.e. $B_{r}(n ; 0) \neq 0$ ) is a root of $B_{r}(n ; x)$, the claim follows from Lemma 3.2 and the definition of $B_{r}(n-1 ; x)$.

We require one final proposition for the proof of Theorem 3.1. Suppose that $f(x)$ (resp. $g(x)$ ) is a degree $n$ (resp. $m$ ) polynomial with roots $x_{1}, \ldots, x_{n}$ (resp. $\left.y_{1}, \ldots, y_{m}\right)$. Furthermore, suppose that

$$
f(x)=\sum_{j=0}^{n} a(j) x^{j} \quad \text { and } \quad g(x)=\sum_{j=0}^{m} b(j) x^{j} .
$$

The resultant $R(f, g)$ of these polynomials satisfies 


$$
\begin{aligned}
R(f, g) & =a(n)^{m} b(m)^{n} \prod_{i=1}^{n} \prod_{j=1}^{m}\left(x_{i}-y_{j}\right)=a(n)^{m} \prod_{i=1}^{n} g\left(x_{i}\right) \\
& =(-1)^{m n} R(g, f) .
\end{aligned}
$$

In particular, notice that if $D(f)$ is the discriminant of $f(x)$, then

$$
R\left(f, f^{\prime}\right)=(-1)^{n(n-1) / 2} D(f) .
$$

Proposition 3.4. If $n \geq 0$, then

$$
\begin{aligned}
R\left(B_{r}(n ; x), B_{r}(n+1 ; x)\right) & =R\left(B_{r}(n+1 ; x), B_{r}(n ; x)\right) \\
& =\prod_{j=1}^{n} h_{r}(j)^{j} c_{r}(j, 0)^{2} .
\end{aligned}
$$

Proof. Since $n(n+1)$ is even, (3.5) implies that the first equality holds for all $n$. If $\alpha$ is a root of $B_{r}(n ; x)$, then Proposition 2.2 implies that

$$
B_{r}(n+1 ; \alpha)=\alpha^{2} h_{r}(n) B_{r}(n-1 ; \alpha) .
$$

Let $\alpha_{1}, \ldots, \alpha_{n}$ be the roots of $B_{r}(n ; x)$ repeated with multiplicity. Since each $B_{r}(j ; x)$ is monic, (3.5) and (3.7) imply that

$$
\begin{aligned}
R\left(B_{r}(n ; x), B_{r}(n+1 ; x)\right) & \\
& =\prod_{j=1}^{n} B_{r}\left(n+1 ; \alpha_{j}\right) \\
& =h_{r}(n)^{n} c_{r}(n, 0)^{2} \prod_{j=1}^{n} B_{r}\left(n-1 ; \alpha_{j}\right) \\
& =h_{r}(n)^{n} c_{r}(n, 0)^{2} R\left(B_{r}(n-1 ; x), B_{r}(n ; x)\right) .
\end{aligned}
$$

Arguing inductively with (3.8), we find that

$$
\begin{aligned}
& R\left(B_{r}(n ; x), B_{r}(n+1 ; x)\right) \\
& \quad=h_{r}(n)^{n} c_{r}(n, 0)^{2} R\left(B_{r}(n-1 ; x), B_{r}(n ; x)\right) \\
& \quad=h_{r}(n)^{n} h_{r}(n-1)^{n-1} c_{r}(n, 0)^{2} c_{r}(n-1,0)^{2} R\left(B_{r}(n-2 ; x), B_{r}(n-1 ; x)\right) \\
& \quad \vdots \\
& \quad=\prod_{j=1}^{n} h_{r}(j)^{j} c_{r}(j, 0)^{2} .
\end{aligned}
$$

Proof of Theorem 3.1. Begin by noticing that $x=0$ is not a zero of $B_{r}(n ; x)$. Now suppose that $\alpha_{1}, \ldots, \alpha_{n}$ are the roots of $B_{r}(n ; x)$ repeated with multiplicity. Since $B_{r}(n ; x)$ is monic, Proposition 3.3 and (3.6) imply 
that

$$
\begin{aligned}
(-1)^{n(n-1) / 2} D_{r}(n) & =R\left(B_{r}(n ; x), B_{r}^{\prime}(n ; x)\right)=\prod_{j=1}^{n} B_{r}^{\prime}\left(n ; \alpha_{j}\right) \\
& =\prod_{j=1}^{n} \frac{\alpha_{j}\left(n-\gamma_{r}+\beta_{r}\right) n}{\left(\alpha_{j}-2\right)\left(2 n+\beta_{r}-1\right)} \cdot B_{r}\left(n-1 ; \alpha_{j}\right) \\
& =\frac{c_{r}(n, 0) n^{n}\left(n-\gamma_{r}+\beta_{r}\right)^{n}}{B_{r}(n ; 2)\left(2 n+\beta_{r}-1\right)^{n}} \cdot \prod_{j=1}^{n} B_{r}\left(n-1 ; \alpha_{j}\right) .
\end{aligned}
$$

By (3.5), this implies that

$$
\begin{aligned}
D_{r}(n)= & (-1)^{n(n-1) / 2}\left(\frac{n\left(n-\gamma_{r}+\beta_{r}\right)}{2 n+\beta_{r}-1}\right)^{n} \cdot \frac{c_{r}(n ; 0)}{B_{r}(n ; 2)} \\
& \times R\left(B_{r}(n ; x), B_{r}(n-1 ; x)\right) .
\end{aligned}
$$

The formula now follows immediately from Proposition 3.4.

4. Proof of Theorem 1.1. In this section, we prove Theorem 1.1 by showing that the $B_{r}(n ; x)$ are $p$-Eisenstein for those $n$ given in Theorem 1.1. This clearly implies that $\widetilde{F}_{k}(j) \in \mathbb{Q}[j]$ is irreducible.

Proof of Theorem 1.1. We re-index $B_{r}(n ; x)$ in this proof to simplify certain calculations, otherwise, we assume the notation from earlier sections. Setting $c_{r}^{\prime}(n, m)=c_{r}(n, n-m)$, we have

$$
\begin{aligned}
B_{r}(n ; x) & =\sum_{m=0}^{n} c_{r}^{\prime}(n, m) x^{n-m} \\
& =\sum_{m=0}^{n} \frac{(-1)^{m}\left(\begin{array}{c}
n \\
m
\end{array}\right)(6 n+r+1) \ldots(6 n+6 m+r-5) x^{n-m}}{\left(3 \gamma_{r}+3\right) \ldots\left(3 m+\left(3 \gamma_{r}-3\right)\right)} .
\end{aligned}
$$

It suffices to show that $p$ divides all coefficients, apart from the leading term, and that $p^{2}$ does not divide the constant term. We present the proof for the case where $r=0$ and explain the additional observations needed for the case $r=4$ along the way. The final two cases, $r=6$ and $r=10$, are closely related to these first two. Our task is simplified by the fact that both 3 and 6 are coprime to $p$, so the arithmetic sequences in the numerator and denominator run through the full set of residue classes modulo $p$.

If $r=0$, let $p=6 d+1$ be a prime congruent to 1 modulo 6 . If $n=$ $(p-1) / 6=d$, then

$$
\begin{aligned}
c_{0}^{\prime}(d, m) & =\frac{(-1)^{m}\left(\begin{array}{c}
d \\
m
\end{array}\right) p(p+6) \ldots(6(n+m)-5)}{2 \cdot 5 \cdot \ldots \cdot(3 m-1)} \\
& =\frac{(-1)^{m}\left(\begin{array}{c}
d \\
m
\end{array}\right) p \ldots(2 p-6(d-m)-5)}{2 \cdot 5 \cdot \ldots \cdot((p-1) / 2-3(d-m)-1)} .
\end{aligned}
$$


The $p$ in the numerator appears in every non-leading coefficient, and no other multiples of $p$ occur in the numerator or denominator. The constant term $c_{0}^{\prime}(d, d)$ is divisible by this single power of $p$, so the series is $p$-Eisenstein. The argument is entirely the same if $r=4, p \equiv 5(\bmod 6)$ and $n=(p-5) / 6$.

Now suppose that $n=(p-1) p^{s} / 6=d p^{s}$, where $s \geq 1$. Since $p^{t} \equiv 1$ $(\bmod 6)$ for any $t$, we can easily describe the multiples of $p$ in the coefficients, $c_{0}^{\prime}\left(d p^{s}, m\right)$

$$
\begin{aligned}
& =\frac{(-1)^{m}\left(\begin{array}{c}
d p^{s} \\
m
\end{array}\right)\left(6 d p^{s}+1\right)\left(6 d p^{s}+7\right) \ldots\left(6\left(d p^{s}+m\right)-5\right)}{2 \cdot 5 \cdot \ldots \cdot(3 m-1)} \\
& =\frac{(-1)^{m}\left(\begin{array}{c}
d p^{s} \\
m
\end{array}\right)\left(6 d p^{s}+1\right) \ldots\left(6 d p^{s}+p^{t}\right) \ldots p^{s+1} \ldots\left(6\left(d p^{s}+m\right)-5\right)}{2 \cdot 5 \cdot \ldots \cdot 2 p^{t} \cdot \ldots \cdot 2 p^{s} \ldots(3 m-1)} .
\end{aligned}
$$

The first multiple of $p^{t}$ occurs in the numerator when $m=\left(p^{t}-1\right) / 6$, and appears in the denominator when $m=\left(2 p^{t}-2\right) / 3=4\left(p^{t}-1\right) / 6$. Every multiple of $p$ in the denominator is cancelled by an equal power in the numerator, and thus $c_{0}^{\prime}(n, m)$ is $p$-integral for all $m$. But $p^{s+1}$ appears as a factor in the numerator when $m \geq\left(p^{s}-1\right) / 6$, and since the denominator factors are bounded above by $p^{s+1} / 2$, there is an extra power of $p$ in the numerator for such $m$. The binomial coefficient $\left(\begin{array}{c}d p^{s} \\ m\end{array}\right)$ is divisible by $p$ for $1 \leq$ $m \leq p^{s}-1$, so $c_{0}^{\prime}(n, m)$ is divisible by $p$ for all $m$. Finally, the numerator of $c_{0}^{\prime}(n, n)$ clearly contains exactly one more power of $p$ than the denominator, so the constant term is not divisible by $p^{2}$.

Next, consider the case of $r=0$ and $p=6 d+5$, and let $c=\left(p^{2}-1\right) / 6=$ $d p+5 d-4$. If $n=c$, then

$$
\begin{aligned}
& c_{0}^{\prime}(c, m) \\
& \quad=\frac{(-1)^{m}\left(\begin{array}{c}
c \\
m
\end{array}\right) p^{2}\left(p^{2}+6\right) \ldots\left(p^{2}+6 p\right) \ldots\left(2 p^{2}-6(c-m)-4\right)}{2 \cdot 5 \cdot \ldots \cdot p \cdot \ldots \cdot 4 p \ldots\left(\left(p^{2}-1\right) / 2-3(c-m)-1\right)} .
\end{aligned}
$$

The first multiple of $p$ in the denominator occurs when $m=2 d+1$, and thus every coefficient is divisible by $p$. Furthermore, there is one extra power of $p$ in the numerator.

If $n=\left(p^{2}-1\right) p^{2 s} / 6=c p^{2 s}$, then

$$
\begin{aligned}
& c_{0}^{\prime}(n, m) \\
& =\frac{(-1)^{m}\left(\begin{array}{c}
n \\
m
\end{array}\right)(6 n+1)(6 n+7) \ldots(6(n+m)-5)}{2 \cdot 5 \cdot \ldots \cdot(3 m-1)} \\
& =\frac{(-1)^{m}\left(\begin{array}{c}
n \\
m
\end{array}\right)(6 n+1) \ldots\left(6 n+p^{2 t}\right) \ldots\left(6 n+5 p^{2 t+1}\right) \ldots p^{2 s+2} \ldots(6(n+m)-5)}{2 \cdot \ldots \cdot 2 p^{2 t} \ldots p^{2 t+1} \ldots p^{2 s+1} \ldots(3 m-1)} .
\end{aligned}
$$

Since $p^{2} \equiv 1(\bmod 6)$, the even powers of $p$ behave exactly like the previous case: the first multiple of $p^{2 t}$ appears as a factor in the numerator when $m=\left(p^{2 t}-1\right) / 6$, and in the denominator when $m=4\left(p^{2 t}-1\right) / 6$. 
However, the situation is more complicated for odd powers of $p$, and the contribution from the binomial coefficients is essential. We use the fact that if $p^{k}$ is the highest power of $p$ that divides $m$, then $\left(\begin{array}{c}c p^{2 s} \\ m\end{array}\right)$ is divisible by $p^{2 s-k}$. The first power of $p^{2 t+1}$ appears in the numerator when

$$
\begin{aligned}
m & =\left(5 p^{2 t+1}-1\right) / 6 \\
& =5(d+1) p(p+1)\left(p^{2 t-2}+\ldots+p^{2}+1\right)+5 d+4,
\end{aligned}
$$

but it appears in the denominator when

$$
\begin{aligned}
m & =\left(p^{2 t+1}-1\right) / 3 \\
& =2(d+1) p(p+1)\left(p^{2 t-2}+\ldots+p^{2}+1\right)+2 d+1 .
\end{aligned}
$$

If $t \leq s-1$ and $m$ is as in (4.5), then the arithmetic sequence in the denominator of $c_{0}^{\prime}(n, m)$ contains $2 t+1$ more powers of $p$ than the numerator. But $\left(\begin{array}{c}c p^{2 s} \\ m\end{array}\right)$ is divisible by $p^{2 s}$, so the coefficient is divisible by $p$. If $m$ increases to the next multiple of $p^{k}$ for some $k$, the factor of $p^{k}$ that is lost in the binomial coefficient appears in the arithmetic sequence of the numerator instead. If $t=s$, and $m$ is as in (4.6), then a factor of $p^{2 s+1}$ appears in the denominator. But $p^{2 s+2}$ appears in the numerator when $m \geq\left(p^{2 s}-1\right) / 6$, and the corresponding term on the denominator is $p^{2 s}$, contributing an overall factor of $p^{2}$. The numerator of $c_{0}^{\prime}(n, n)$ contains exactly one extra power of $p$, since every term on the denominator is strictly less than $p^{2 s+2}$, so the series is $p$-Eisenstein.

The argument is similar if $r=4, p=6 d+5$ and $n=c p^{2 s+1}$, with (4.4) replaced by

$$
\begin{aligned}
& c_{4}^{\prime}(n, m) \\
& =\frac{(-1)^{m}\left(\begin{array}{c}
n \\
m
\end{array}\right)(6 n+5)(6 n+11) \ldots(6(n+m)-1)}{4 \cdot 7 \cdot \ldots \cdot(3 m+1)} \\
& =\frac{(-1)^{m}\left(\begin{array}{c}
n \\
m
\end{array}\right)(6 n+5) \ldots\left(6 n+5 p^{2 t}\right) \ldots\left(6 n+p^{2 t+1}\right) \ldots p^{2 s+3} \ldots(6(n+m)-1)}{2 \cdot \ldots \cdot p^{2 t} \cdot \ldots \cdot 2 p^{2 t+1} \ldots p^{2 s+2} \ldots(3 m+1)} .
\end{aligned}
$$

The key difference is that all of the exponents have been shifted by one because the factors in the numerator of $c_{4}^{\prime}(n, m)$ are all congruent to 5 modulo 6 instead of 1 modulo 6 , and the factors in the denominator are congruent to 1 modulo 3 instead of 2 modulo 3 .

Equation (4.1) shows that if $r=6$, then the coefficients are nearly the same as in the case $r=0$ through the relation

$$
c_{6}^{\prime}(n, m)=\frac{c_{0}^{\prime}(n, m)(6(n+m)+1)}{6 n+1} .
$$

Thus, if $B_{0}(n ; x)$ is $p$-Eisenstein for some $n$ and $p$ does not divide $6 n+1$ or $12 n+1$, then $B_{6}(n ; x)$ is $p$-Eisenstein for the same $n$. The only exceptions occur when $6 n+1=p$ if $p \equiv 1(\bmod 6)$ and when $6 n+1=p^{2}$ if $p \equiv 5$ 
$(\bmod 6)$. In these cases, $(4.2)$ and $(4.3)$ show that the first factor in the arithmetic sequence of the numerator of $c_{0}^{\prime}(n, m)$ is $p$ or $p^{2}$. The analogous case for $r=6$ occurs if $6 n+7=p$ or $6 n+7=\widetilde{p}^{2}$. When $p=7$, this is impossible (since $n \geq 1$ ), so the family of allowable $n$ values starts at a higher power; $n=(7-1) 7^{s} / 6=7^{s}$ for $s \geq 1$.

Similarly, if $r=10$, then there is an analog to (4.8) that shows that $B_{10}(n ; x)$ is $p$-Eisenstein for nearly all of the same $n$ as $B_{4}(n ; x)$; the differences are described in the theorem.

Remark. Arguing as above, it can be shown that the $B_{r}(n ; x)$ which are $p$-Eisenstein for a prime $p \geq 5$ are precisely those polynomials identified by Theorem 1.1.

5. Proof of Theorem 1.2. The polynomials $\widetilde{F}_{k}(j)$ in Theorem 1.2 are irreducible over $\mathbb{Q}$ by Theorem 1.1. To prove Theorem 1.2 , it suffices to show that the discriminant $D_{r}(n)$ of $B_{r}(n ; x)$, for the associated $n$, is not a square of a rational number. Through a careful analysis of the discriminant formula found in Section 3, we prove that the power of $p$ dividing $D_{r}(n)$ is odd. Before beginning the proof, we present a pair of technical lemmas.

Lemma 5.1. Given an integer $k$ and $-k<l<k$, let $p$ be an odd prime such that $p \equiv 1(\bmod k)$. Define $\lambda \equiv l(\bmod k)$ such that $-k \leq \lambda \leq-1$. If $n=d p^{s}$, where $d k<p$ and $\left(k d p^{s-1}+\lambda\right) p \leq\left(d p^{s}-1\right) k+l$, then the product

$$
P=\prod_{j=1}^{n-1}(k j+l)^{j}
$$

satisfies

$$
\operatorname{ord}_{p}(P)=\frac{d p^{s}}{2}\left(s+d\left(\frac{p^{s}-1}{p-1}\right)\right)-\frac{l d}{k}\left(\frac{p^{s}-1}{p-1}\right)+\frac{\lambda s d p^{s}}{k} .
$$

Proof. The formula is found by directly counting the powers of $p$ in $P$. Since $p^{t} \equiv 1(\bmod k)$ for any $t$, the multiples of $p^{t}$ that appear as factors in $P$ have the form $\left(m p^{t}\right)^{\left(m p^{t}-l\right) / k}$, where $m \equiv l(\bmod k)$ and $0 \leq m \leq$ $\left(k d p^{s-t}+\lambda\right) p^{t}$. Define $\zeta$ such that $0 \leq \zeta \leq k-1$ and $\zeta \equiv l(\bmod k)$ (note that if $l \geq 0$, then $\zeta=l$ and $\lambda=l-k$; if $l<0$, then $\zeta=l+k$ and $\lambda=l$ ). Then the product can be written

$$
\begin{aligned}
P= & (k+l)^{1} \ldots(\zeta p)^{(\zeta p-l) / k} \ldots\left(\zeta p^{t}\right)^{\left(\zeta p^{t}-l\right) / k} \ldots \\
& \times\left(\left(k d p^{s-t}+\lambda\right) p^{t}\right)^{\left(\left(k d p^{s-t}+\lambda\right) p^{t}-l\right) / k} \ldots\left(\left(d p^{s}-1\right) k+l\right)^{d p^{s}-1} .
\end{aligned}
$$

To find the total exponent of $p$ in $P$, add the exponents appearing on each multiple of $p$, then add the exponents on multiples of $p^{2}$, and continue 
up to multiples of $p^{s}$. This is given by the sum

$$
\begin{aligned}
& \frac{\zeta p-l}{k}+\frac{(k+\zeta) p-l}{k}+\ldots+\frac{\zeta p^{2}-l}{k}+\ldots+ \frac{\zeta p^{s}-l}{k}+\ldots+\frac{\left(k d p^{s-1}+\lambda\right) p-l}{k} \\
&+\frac{\zeta p^{2}-l}{k}+\ldots+\frac{\zeta p^{s}-l}{k}+\ldots+\frac{\left(k d p^{s-2}+\lambda\right) p^{2}-l}{k} \\
& \vdots \\
&+\frac{\zeta p^{s}-l}{k}+\ldots+\frac{(k d+\lambda) p^{s}-l}{k} .
\end{aligned}
$$

The final condition in the statement of the theorem ensures that all of these multiples of $p$ actually appear in $P$.

Each line is an arithmetic sequence that has been uniformly shifted; the $t$ th line contains $d p^{s-t}$ terms. The sum is thus

$$
\begin{aligned}
\frac{(\zeta-\lambda) p}{k}+\frac{(k+\zeta-\lambda) p}{k}+\ldots & +\frac{\zeta p^{s}-\lambda p}{k}+\ldots+\frac{\left(k d p^{s-1}+\lambda-\lambda\right) p}{k}-\frac{l}{k} d p^{s-1}+\frac{\lambda p}{k} d p^{s-1} \\
& \vdots \\
& +\frac{(\zeta-\lambda) p^{s}}{k}+\ldots+\frac{(k d+\lambda-\lambda) p^{s}}{k}-\frac{l}{k} d+\frac{\lambda p^{s}}{k} d .
\end{aligned}
$$

This is simplified by the fact that $\zeta-\lambda=k$, giving

$$
\begin{aligned}
p+2 p+\ldots & +d p^{s}-\frac{l}{k} d p^{s-1}+\frac{\lambda p}{k} d p^{s-1} \\
& \vdots \\
& +p^{s}+\ldots+d p^{s}-\frac{l}{k} d+\frac{\lambda p^{s}}{k} d .
\end{aligned}
$$

Summing the arithmetic and geometric progressions and collecting like terms gives the formulas.

Lemma 5.2. Given $k$, and $-k<l<k$, let $p$ be an odd prime such that $p=d k+r$ with $0 \leq r \leq k-1$ and $r^{2} \equiv 1(\bmod k)$. Let $\lambda \equiv l(\bmod k)$ be defined such that $-k \leq \lambda \leq-1$, and let $\kappa=\lambda+k$. Also, let $\bar{\lambda} \equiv r \kappa(\bmod k)$ such that $\bar{\lambda}$ lies in the same range as $\lambda$, and define $\bar{\kappa}=\bar{\lambda}+k$. If $n=c p^{2 s}$, where $c k<p^{2}$ and $\left(k c p^{2 s-1}+\bar{\lambda}\right) p \leq k c\left(p^{2} s-1\right)+l$, let $\bar{d}$ be the largest integer such that $(k \bar{d}+\bar{\kappa}) p^{2 s+1}<k\left(c p^{2 s}-1\right)+l$. Then the product

$$
P=\prod_{j=1}^{n-1}(k j+l)^{j}
$$

satisfies 


$$
\begin{aligned}
& \operatorname{ord}_{p}(P) \\
& =\frac{c p^{2 s}}{2}\left(2 s+c\left(\frac{p^{2 s}-1}{p-1}\right)\right)-\frac{l c}{k}\left(\frac{p^{2 s}-1}{p-1}\right)+\frac{2 s c p^{2 s}(\lambda+\bar{\lambda}) / 2}{k} \\
& -\frac{l \bar{d}}{k}+\frac{\bar{\lambda} \bar{d} p^{2 s+1}}{k}+\frac{p^{2 s+1} \bar{d}(\bar{d}+1)}{2} .
\end{aligned}
$$

The proof of Lemma 5.2 follows as in the proof of Lemma 5.1, and it is omitted for brevity.

Proof of Theorem 1.2. Suppose that $p \geq 5$ is a prime, and that $n$ and $r$ satisfy the conditions of Theorem 1.2. Recall from Theorem 3.1 the formula for the discriminant:

$$
\begin{aligned}
D_{r}(n)= & (-1)^{n(n-1) / 2}\left(\frac{n\left(n-\gamma_{r}+\beta_{r}\right)}{2 n+\beta_{r}-1}\right)^{n} \cdot \frac{c_{r}(n, 0)}{B_{r}(n ; 2)} \\
& \times \prod_{j=1}^{n-1} h_{r}(j)^{j} c_{r}(j, 0)^{2} .
\end{aligned}
$$

We will show that $p$ occurs as a factor of $D_{r}(n)$ with odd multiplicity by presenting careful arguments in the case where $r=0$ and noting the slight differences in proving the other cases.

Suppose that $r=0, p=12 d^{\prime}+1$ and $n=d p^{s}$, where $d=2 d^{\prime}$. The first term in (5.3) is

$$
\left(\frac{n\left(n-\gamma_{0}+\beta_{0}\right)}{2 n+\beta_{0}-1}\right)^{n}=\left(\frac{n(n-2 / 3+1 / 6)}{2 n+16-1}\right)^{n}=\left(\frac{n(6 n-3)}{12 n-5}\right)^{n} .
$$

Since $p \geq 13$, the only multiples of $p$ appear in $n$ itself, and the total product of factors of $p$ is $\left(p^{s}\right)^{d p^{s}}$. If $r=6, p=12 d^{\prime}+7, s \geq 1$, and $n=d p^{s}$, where $d=2 d^{\prime}+1$, then the analogous term in $(5.3)$ is $(n(6 n+3) /(12 n+1))^{n}$, which also has the factors $\left(p^{s}\right)^{d p^{s}}$. When $s=0$, there are no powers of $p$ in the $r=0$ case, corresponding to the case where $n=(p-7) / 6$ if $r=6$.

We showed in Section 4 that the monic polynomial $B_{r}(n ; x)$ is $p$-Eisenstein for any value of $r$, so that $p$ divides every non-leading coefficient, and $p^{2}$ does not divide the constant term $c_{r}(n, 0)$. Thus $c_{r}(n, 0)$ is divisible by exactly one multiple of $p$, and $B_{r}(n ; 2)$ contains no power of $p$, for $B_{r}(n ; 2)$ $\equiv 2^{n}(\bmod p)$.

Now we turn to the product factors in (5.3). Since we wish to show that it is not a perfect square, we can ignore the factors of the form $c_{0}(j, 0)^{2}$. We could also remove all of the square powers from the remaining terms and just consider the exponents modulo 2 , but our lemmas count the prime factors when all of the factors are present. Since $p$ is prime to 9 , we ignore 
the factor of 9 in $h_{r}(j)$ throughout the rest of the proof. We have

$$
\prod_{j=1}^{n-1} h_{0}(j)^{j}=\prod_{j=1}^{n-1}\left(\frac{-9 j(2 j-1)(12 j+7)}{(3 j+2)(6 j+1)(12 j-5)}\right)^{j} .
$$

Since $n=d p^{s}$,

$$
\prod_{j=1}^{n-1}\left(\frac{12 j+7}{12 j-5}\right)^{j}=\left(12 d p^{s}-5\right)^{d p^{s}-1} \prod_{j=1}^{d p^{s}-1} \frac{1}{12 j-5},
$$

and the factors of $p$ multiply to $p^{-d\left(p^{s}-1\right) /(p-1)}$. In the case where $r=6$, $p=12 d^{\prime}+7$ and $n=d p^{s}$, the analog of (5.4) is easily found, and the corresponding formula for (5.5) now depends on the parity of $s$.

Lemma 5.1 counts the factors of $p$ in the remainder of (5.4), since $p=12 d^{\prime}+1 \equiv 1(\bmod 2,3,6,12)$. For example, this lemma states that the product $\prod_{j=1}^{d p^{s}-1}(3 j+2)^{j}$ contains

$$
\frac{2 d^{\prime} p^{s}}{2}\left(s+2 d^{\prime} \frac{p^{s}-1}{p-1}\right)-\frac{2\left(2 d^{\prime}\right)}{3} \cdot \frac{p^{s}-1}{p-1}-\frac{s\left(2 d^{\prime}\right) p^{s}}{3}
$$

total factors of $p$. Thus the parity of the total power of $p$ in $D_{0}(n)$ is found by adding $1+s d p^{s}$ to the factors in (5.4), giving

$$
\begin{aligned}
1+s d p^{s}+d \frac{p^{s}-1}{p-1} & \left(0+\frac{1}{2}+\frac{2}{3}+\frac{1}{6}-1\right)+s d p^{s}\left(-1-\frac{1}{2}+\frac{1}{3}+\frac{5}{6}\right) \\
= & \frac{d}{3} \frac{p^{s}-1}{p-1}-\frac{s d p^{s}}{3}+1+s d p^{s}=1+\frac{d}{3}\left(2 s p^{s}+\frac{p^{s}-1}{p-1}\right) .
\end{aligned}
$$

Since $d=2 d^{\prime}$ is even, this power is clearly odd. The argument is similar if $r=6$ and $p=12 d^{\prime}+7$, although it must be argued that Lemma 5.1 can be applied to $\prod_{j=1}^{n-1}(6 j+7)^{j}$, since the final factor is not divisible by $p$ and can be removed.

Now suppose that $r=0, p=6 d+5$, and $n=c p^{2 s}$. The case $p=5$ must be treated separately, since the first factors in (5.3) are

$$
\left(\frac{n(6 n-3)}{12 n-5}\right)^{n}=\left(\frac{c \cdot 5^{2 s}\left(6 c \cdot 5^{2 s}-3\right)}{5\left(12 c \cdot 5^{2 s-1}-1\right)}\right)^{c \cdot 5^{2 s}} .
$$

The product of all of the factors of 5 is $5^{(2 s-1) c \cdot 5^{2 s}}$, and there is one multiple of 5 in $c_{0}(n, 0)$. Otherwise, if $p \neq 5$, the factors give $p^{2 s c p^{2 s}}$. If $r=4$ or $r=10$ and $n=c p^{2 s+1}$ then the product is $p^{(2 s+1) c p^{2 s+1}}$.

We now use Lemma 5.2 to count the multiples of $p$ in (5.4), for $p^{2}$ $\equiv 1(\bmod 2,3,6)$. First, consider the case where $d$ is even. There is a potential concern when $p=5$, for the initial terms in (5.3) only contain $1+(2 s-1) c p^{2 s}$ powers of $p$. However, the formula that we find will still be valid, for one of the conditions of Lemma 5.2 is not met by the product 
$\prod_{j=1}^{n-1}(12 j-5)^{j}$ when $p=5$. In fact, the final multiple of $p$ that is counted by the lemma occurs when $j=n=c p^{2 s}$, and hence it is not actually contained in the product. This term appears on the denominator of (5.4), so the factors of $p$ cancel exactly. If we write $c=\left(p^{2}-1\right) / 6=d p+5 d+4$, it is clear that $\bar{d}=d+1$ exactly when $k(5 d+4) \geq \bar{\kappa} p$, and otherwise $\bar{d}=d$. After simplification and eliminating terms which are clearly even, the total power of $p$ is thus

$$
2+s(d+1)(p-1) p^{2 s}+2(d+1) \frac{p^{2 s}-1}{3}+\frac{d\left(p^{2 s+1}+1\right)}{3}+\frac{p^{2 s+1}+1}{6} .
$$

Since $d$ is even, every term except the final one is even. The final term is odd, since

$$
\begin{aligned}
\frac{p^{2 s+1}+1}{6} & =(d+1)\left(p^{2 s}-p^{2 s-1}+\ldots-p+1\right) \\
& \equiv 1-1+\ldots+1-1+1 \equiv 1(\bmod 2) .
\end{aligned}
$$

If $d$ is odd, then Lemma 5.2 gives the same formulas, and the congruence properties of $p$ again show that the total exponent is odd.

Finally, if $r=4$ or $r=10$ and $n=c p^{2 s+1}$, the argument is similar, although the formulas become more complicated. Lemma 5.2 nearly applies, with $2 s$ replaced by $2 s+1$ throughout, but the odd exponent changes the relative weighting of $\bar{\kappa}$ and $\bar{\lambda}$ in (5.2). There are $s+1$ different odd powers of $p$ so $\bar{\lambda}$ appears in the proportion $(s+1) /(2 s+1)$, but there are only $s$ even powers, so $\lambda$ makes up the remaining $s /(2 s+1)$ fraction. Also, since the highest power is $p^{2 s+2}$, the $\bar{\lambda}$ in (5.2) is replaced by $\lambda$. For example, if $r=4, p=6 d+5$ with $d$ even, and $n=c p^{2 s+1}$, then $\prod_{j=1}^{n-1}(6 j+5)^{j}$ contains

$$
\begin{aligned}
\frac{c p^{2 s+1}}{2}\left(2 s+1+c \frac{p^{2 s+1}-1}{p-1}\right) & -\frac{5 c}{6} \cdot \frac{p^{2 s+1}-1}{p-1}-\frac{5(s+1) c p^{2 s+1}}{6}-\frac{s c p^{2 s+1}}{3} \\
& -\frac{5 d}{6}-\frac{d p^{2 s+2}}{6}+\frac{p^{2 s+2} d(d+1)}{2}
\end{aligned}
$$

powers of $p$.

In all of the cases given in Theorem 1.2, the total exponent of $p$ is odd, and so $B_{r}(n ; x)$ is an irreducible polynomial whose Galois group is not a subgroup of $A_{d_{k}}$.

\section{References}

[AAR] G. E. Andrews, R. Askey and R. Roy, Special Functions, Encyclopedia Math. Appl. 71, Cambridge Univ. Press, 1999.

[K] M. Kaneko, On the zeros of certain modular forms, in: Number Theory and its Applications (Kyoto, 1997), S. Kanemitsu and K. Győry (eds.), Dev. Math. 2, Kluwer, 1999, 193-197. 
[K-N] M. Kaneko and N. Niiho, private communication.

[K-Z] M. Kaneko and D. Zagier, Supersingular $j$-invariants, hypergeometric series, and Atkin's orthogonal polynomials, in: Computational Perspectives on Number Theory (Chicago, IL, 1995), D. A. Buell and J. T. Teitelbaum (eds.), AMS/IP Stud. Adv. Math. 7, Amer. Math. Soc., 1998, 97-126.

[R-S] F. K. C. Rankin and H. P. F. Swinnerton-Dyer, On the zeros of Eisenstein series, Bull. London Math. Soc. 2 (1970), 169-170.

Department of Mathematics

University of Wisconsin

Madison, WI 53706, U.S.A.

E-mail: mahlburg@math.wisc.edu

ono@math.wisc.edu 